\title{
Sources of sedimentary PAHs in tropical Asian waters: differentiation between pyrogenic and petrogenic sources by alkyl homolog abundance
}

\begin{abstract}
We collected surface sediment samples from 174 locations in India, Indonesia, Malaysia, Thailand, Vietnam, Cambodia, Laos, and the Philippines and analyzed them for polycyclic aromatic hydrocarbons (PAHs) and hopanes. PAHs were widely distributed in the sediments, with comparatively higher concentrations in urban areas (XPAHs: $\sim 1000$ to $\sim 100000 \mathrm{ng} / \mathrm{g}$ dry) than in rural areas ( $\sim 10$ to $\sim 100 \mathrm{~g}$-dry), indicating large sources of PAHs in urban areas. To distinguish petrogenic and pyrogenic sources of PAHs, we calculated the ratios of alkyl PAHs to parent PAHs: methylphenanthrenes to phenanthrene (MP/P), methylpyrenes + methylfluoranthenes to pyrene + fluoranthene $(\mathrm{MPy} / \mathrm{Py})$, and methylchrysenes + methylbenz[a]anthracenes to chrysene + benz[a]anthracene $(\mathrm{MC} / \mathrm{C})$. Analysis of source materials (crude oil, automobile exhaust, and coal and wood combustion products) gave thresholds of $\mathrm{MP} / \mathrm{P}=0.4, \mathrm{MPy} / \mathrm{Py}=0.5$, and $\mathrm{MC} / \mathrm{C}=1.0$ for exclusive combustion origin. All the combustion product samples had the ratios of alkyl PAHs to parent PAHs below these threshold values. Contributions of petrogenic and pyrogenic sources to the sedimentary PAHs were uneven among the homologs: the phenanthrene series had a greater petrogenic contribution, whereas the chrysene series had a greater pyrogenic contribution. All the Indian sediments showed a strong pyrogenic signature with MP/P å 0.5, MPy/Py å 0.1, and MC/C å 0.2 , together with depletion of hopanes indicating intensive inputs of combustion products of coal and/or wood, probably due to the heavy dependence on these fuels as sources of energy. In contrast, sedimentary PAHs from all other tropical Asian cities were abundant in alkylated PAHs with MP/P å 1ї 4, MPy/Py å 0.3ï 1, and MC/C å 0.2ï 1.0, suggesting a ubiquitous input of petrogenic PAHs. Petrogenic contributions to PAH homologs varied among the countries: largest in Malaysia whereas inferior in Laos. The higher abundance of alkylated PAHs together with constant hopane profiles suggests widespread inputs of automobile-derived petrogenic PAHs to Asian waters.
\end{abstract}

Keyword: Polycyclic aromatic hydrocarbons; Water pollutants; Chemical; Sediment; Water pollution 